\title{
SISTEMA "KILLER" EN CEPAS DE CANDIDA ALBICANS. PARTE II.
}

\author{
Hortensia María Magaro, Marisa Susana Biasoli \\ \& Blanca Julieta Corallini de Bracalenti. \\ Departamento de Microbiologia (Area Micología). \\ Facultad de Ciencias Bioquímicas y Farmacéuticas. \\ Universidad Nacional de Rosario. \\ Suipacha 531. Rosario (2000). Santa Fé República Argentina.
}

Palabras clave : Sistema Killer, Candida albicans, epidemiología.

Key Words: Killer system, Candida albicans, epidemiology.

\section{RESUMEN}

Nueve levaduras diferentes con capacidad killer fueron enfrentadas a cepas de $\mathbf{C}$. albicans.

Otro sistema killer (SKA) obtenido de levaduras de la Micoteca del Departamento de Microbiologia de la Facultad de Ciencias Bioquímicas y Farmacéuticas de la Universidad Nacional de Rosario, fue también enfrentado con cepas de C. albicans aisladas en la República Argentina.

Según el modelo de inhibición observado en las cepas, éstas fueron codificadas en tripletes, adjudicándole a cada una de ellas un número de código, que permita posteriormente establecer los tipos killer.

Los números de código obtenidos por ambos métodos estudiados sobre 50 cepas de C. albicans fueron idénticos en el $90 \%$ de los casos, el tipo killer más frecuente fue el 111; lo cual sugiere que podria tratarse de las mismas toxinas producidas por cepas distintas, hecho que debe ser comprobado.

El objetivo de este trabajo fue comparar dos sistemas (SKI y SKA) sobre cepas de C. albicans. La metodología empleada fue la de Polonelli y col.

\section{INTRODUCCION}

$\mathrm{C}$ albicans es la levadura más frecuentemente aislada de materiales clínicos como comensal o patógeno, sin embargo, estudios detallados de sus fuentes y modos de transmisión como agente etiológico han sido escasos en ausencia de un método que permita la subdivisión fina de cepas o biotipos dentro de especies (8).

\section{SUMMARY}

[ Killer System in Candida albicans strains, Part II]

Nine different yeasts having "killer" capability compared with $\mathbf{C}$. albicans strains.

Another "killer" system (SKA) obtained from our culture collection (Departamento de Microbiologia de la Facultad de Ciencias Bioquímicas y Farmacéuticas de la Universidad Nacional de Rosario) was also compared with $\mathbf{C}$. albicans strains isolated by us.

According to the observed pattern of inhibition in the strains, these were codified in triplets with a code number, for each one of them to establish killer types later.

The code numbers obtained through the two studied methods on 50 strains of $\mathbf{C}$. albicans were the same in a $90 \%$ of all cases being the killer type 111 , the most frequent. This suggests that different strains may produce the same toxins, however; this fact must be proved.

The aim of this work is to compare the two methods, SKI and SKA, on strains of C. albicans. The methodology, used was Polonelli's et al. technique.

El método más adecuado está basado en la división de las cepas de $\mathbf{C}$. albicans aisladas, en dos grupos serológicos (A y B) (5).

También como los dos serotipos de C. albicans hay algunos otros ejemplos publicados de variación de cepas dentro de especies, incluyendo diferencias en la morfología de la colonia sobre agar malta (2), virulencia en animales de laboratorio (7, 11), resistencia a 5 fluorcitosina $(3,4,13)$, secreción de proteinasa (12) y capacidad para utilizar algunos 
azúcares como única fuente de carbono para el crecimiento (14), entre otros.

Polonelli y col. iniciaron el estudio con fines epidemíogicos del fenómeno killer en levaduras, el cual permitio el desarrollo de un sistema simple (sistema killer) util para diferenciar cepas de levaduras oportunistas, incluyendo a Candida albicans, dentro de las especies $(6,9,10)$.

La búsqueda de un sistema killer similar al hallado por Polonelli y col., en cepa de la Micoteca del Area Micología del Departamento de Microbiologia de la Facultad de Ciencias Bioquímicas y Farmacéuticas de la Universidad Nacional de Rosario nos permitio lograr uno que denominamos SKA (Sistema Killer Argentino).

El sistema killer de Polonelli y col. lo denominamos SKI (Sistema Killer Italiano).

El objetivo de este trabajo fue comparar esos dos sistemas (SKI y SKA) sobre cepas de C. albicans para evaluarlo posteriormente como posible marcador epidemiológico sobre cepas de levaduras (C. albicans y Candida spp.).

\section{MATERIALES Y METODOS}

Se trabajó con 50 cepas de C. albicans procedentes de diferentes materiales clínicos que se procesaron en el Laboratorio Asistencial del Area de Micología del Departamento de Microbiología de la Facultad de Ciencias Bioquímicas y Farmacéuticas de la Universidad Nacional de Rosario.

Las 50 cepas se probaron frente al sistema killer propuesto por Polonelli y col. (9) y el desarrollado por Magaró y col. (Parte I).

Las nueve cepas seleccionadas para formar el SKA correspondieron a:

1) Candidia albicans, CMUR № 100-73

2) Candicua albicans, CMUR No 101-73

3) Canoida albicans, CMUR № 100-74

4) Candida albicans, CMUR N 103-74

5) Candida albicans, CMUR N2 104-74

6) Candida guilliermondii, CMUR № 108-74

7). Candida catemulata, CMUR Ne 115-71

8) Hansenula anomala, FFBBA № 264

9) Candida ukilis, CMUR No 161-74

CMUR: Colección Micología Universidad Rasario FFBBA: Facultad Farmacia y Bioquímica Buenos Aires

\section{RESULTADOS}

Los resultados obtenidos con cada una de las $\mathbf{5 0}$ cepas de C. albicans y los dos sistemas killer (SKI y SKA) fueron agrupados en tripletes (9), de modo tal que si una cepa de $C$. albicans fue sensible a las nueve levaduras killer le correspondió el código 111.

En la Tabla № 1, se indica el origen de las cepas de $\mathbf{C}$. albicans aisladas.

Los números de código obtenidos con las 50 cepas estudiadas y probadas con los sistemas SKI y SKA se señalan en la Tabla № 2. Los resultados de la misma se indican en el siguiente cuadro comparativo entre los sistemas SKI y SKA, expresándose el porcentaje de $\mathbf{C}$. albicans que coincidieron o no con ambos sistemas

\begin{tabular}{lr} 
Coincidencia entre & \\
ambos sistemas & $\mathbf{9 0 , 0 \%}$ \\
No coincidencias & $10,0 \%$ \\
\cline { 2 - 2 } Total & $100,0 \%$
\end{tabular}

Número de cepas de C. albicans

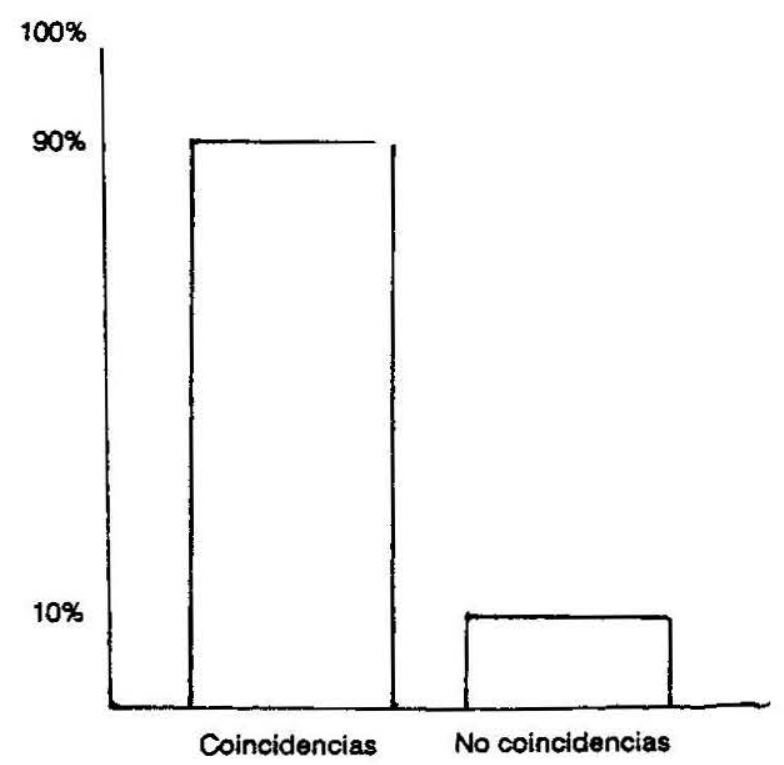

\section{DISCUSION Y CONCLUSIONES}

La Tabla № 1 muestra que la fuente principal del aislamiento de las 50 cepas de $\mathbf{C}$. albicans fue flujo vaginal (39), siguiéndole con menor frecuencia orina, esputo, etc. 
Tabla NP 1

Origen de las 50 cepas de C. Albieans aisladas.

\begin{tabular}{|lr|}
\hline Material & $\begin{array}{r}\text { Nomero de } \\
\text { cepas } \\
\text { aisladas }\end{array}$ \\
\hline Flujo vaginal & 39 \\
Orina & 4 \\
Exputo & 3 \\
Region anal & 1 \\
Unta & 1 \\
\hline Mucosa yugal & 1 \\
\hline Total de cepas & 2 \\
\hline
\end{tabular}

Estas cepas so enfrentaron con los dos sistemas biller en estudio (SKI y SKA) obtenićndose los números de códigos que figuran en la Tabla $\mathrm{N}^{\circ} \mathbf{2}$, pudiendose observar que a 45 cepas de C. albleans le correspondio el tipo killer 111. Estos resultados muestran que en la población estudiada, ese es el tipo prevalente para ambos sistemas.

La Tabla No 2 muestra que las respuestas a ambos sistemas de las levaduras estudiadas fue la misma en un $90 \%$ ( $45 \mathrm{C}$. albleans) y no hubo coincidencias en un $10 \%$ ( 5 C. albleans). Con respecto a esto oltimo, en una sola cepa ie apreció una discrepancia de 2 levaduras killer, mientras que en el resto la diferencia fue de una sola levadura.
Tabla No 2

Número de código de las 50 cepas de C. albleans obtenidos con el SKI y el SKA.

\begin{tabular}{|ccc|}
\hline $\begin{array}{c}\text { Número de } \\
\text { codigo SKI }\end{array}$ & $\begin{array}{c}\text { Número de } \\
\text { codigo SKA }\end{array}$ & $\begin{array}{c}\text { Namero } \\
\text { de cepas }\end{array}$ \\
\hline 111 & 111 & 45 \\
111 & 112 & 2 \\
111 & 115 & 1 \\
241 & 332 & 1 \\
411 & 231 & 1 \\
\hline TOTAL & & 50 \\
\hline
\end{tabular}

La concordancia en las respuestas del $90 \%$ de las levaduras probadas para ambos sistemas solo demuestran la sensibilidad de las mismas al efecto killer de las levaduras componentes del SKI y del SKA. Los resultados obtenidos hasta el momento no permiten establecer similitudes entre ambos equipos tipificadores hasta no demostrar que las toxinas producidas por cada una de las cepas killer sea la misma.

Segun Morace y col. (6), las levaduras no patógenas son resistentes a las cepas killer, mientras que las patógenas muestran mayor sensibilidad. Todas las cepas analizadas por nosotros provenfan de materiales clínicos de pacientes con patologias comprobadas, por lo cual, los resultados obtenidos demuestran una estrecha relación entre sensibilidad al efecto killer, patogenicidad y procedencia anatómica de la población estudiada.

\section{REFERENCIAS}

1. Bevn, EA \& Makower, M. (1963). The physiological basis of the kller charecter in yeast. In. S. J. Geerts ed. Genetic Today. XIth Intemational Congress on Genetics, Vol. 1, 202-203.
2. Brown-Thomeen, J. (1968), Variability in Candida albicans (Robin) Berthout, J. Studies on morphology and blochemical activity. Hereditas, $60,355-398$. 
3. Drouhet, E; Mercier-Soucy, L. \& Montplaisir, S. (1975). Sensibilite et resistance des levures pathogenes aux Sfluoropyrimidines I. Relation entre les/phenotypes de is redittance a la Sulluorocytosine, le sorotupe de Cardida albicans et Yecologle de differentes lespeces de Candida d'origine humaine. Annales de Microbiologie, 126 BM, 2539.

4. Hamilton-Miller, J.M.T. (1972) A comparative in vitro study of amphotericin B, clotrimazole and S-fluorocytocine against clinically isolated yeasts. Sabouraudia 10, 276-283.

5. Hasenclever, H.F. \& Mitchell, W.O. (1961). Antigenit studies of Candida I. Observation of two antigenic groups in Candida albicans. Joumal of Bacteriology, 82, 570-573.

6. Morace, G.; Archibusacei, G.; Sestito, M. \& Polonelli, $\mathbf{L}^{t}$ (1984). Strain differentiation of pathogenic yeasts by the killer system. Mycopathologia, 84, 81-85.

7. Mourad, S. \& Friedman, L. (1961). Pathogenicity of Candida. Journal of Bacteriology, 81, 550-556.

8. Odds F.C. (1979). Candida and Candidosis Leicesten Leicester University Press.$$
\text { ........ }
$$

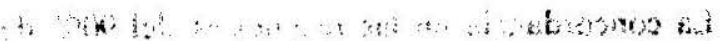

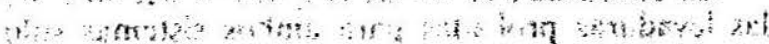

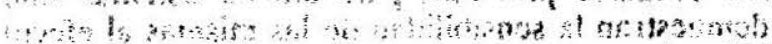

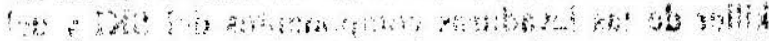

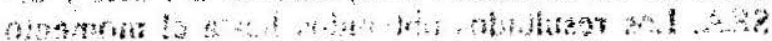

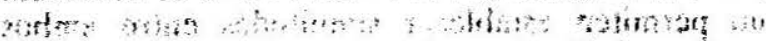

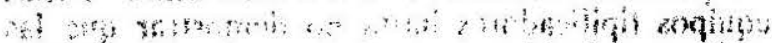

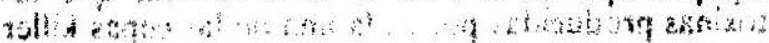$$
\text { rision of at: }
$$

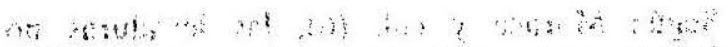

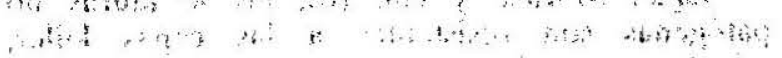

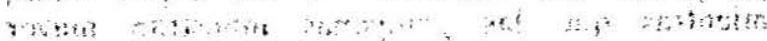

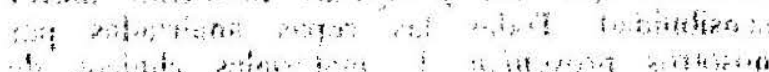

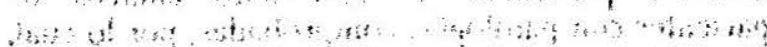

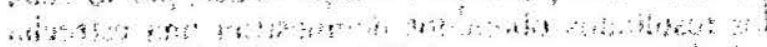

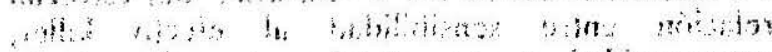

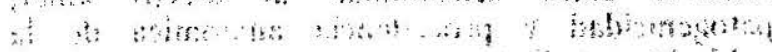

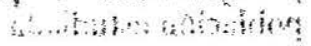

9. Polonelli, La; A"rchịbusqceci, C.: Sestito, M. \& Morace, G. (1983). Killer system: a simple method for differentiating Candida albicans strain Joumal of Clinical Microbiology, $17,774-780$.

10. Polonelli, L; Castagnola, M.; Rossetti, D.V. \& Morace, G. (1985). Use of killer toxins for computez-aided differentiation of Candida albicans strains. Mycopathologia, $91,175-179$.

11. Sandula, J.; Kockova-Kratochuilova, A. \& Zamecnikova, M. (1963). Genus Candida Berkhout II. Pathogenicity of the species Candida albicans (Robin) Berkhout. Folia Microbiológica, 8, 313-317.

12. Staib, F. (1965). Serum-proteins as nitrogén source for yeast-like fungi. Sabouraudia, 4, 187-193.

13. Steer, P.L.; Marks, M.I.; Klite, P.D. \& Eickhoff, T.C. (1972). S-fluorocytosine: an oral antifungal compound. Annals of Internal Medicine, 76, 15-22.

14. Van Der Walt \& Yarrow, D. (1984). The Yeasts ( Elsevier Publishers B.V. Amsterdam

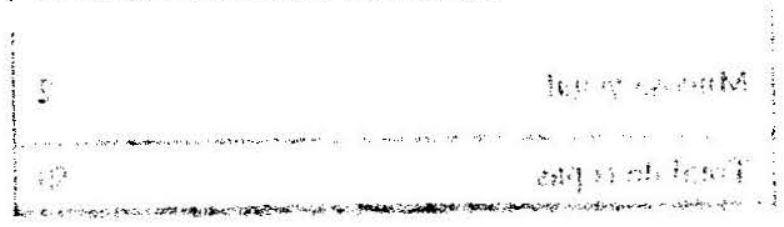

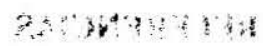

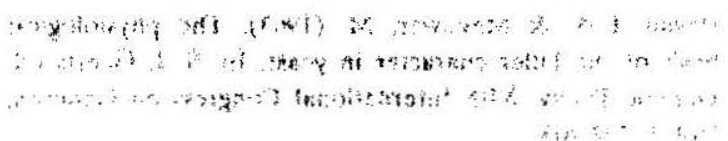

